\title{
Review of Rabies Preventions and Control
}

\author{
Chernet Balcha, Nejash Abdela \\ College of Agriculture and Veterinary Medicine, School of Veterinary Medicine, Jimma University, Ethiopia
}

\begin{tabular}{l} 
Article Info \\
\hline Article history: \\
Received Sep 18, 2017 \\
Revised Nov 23, 2017 \\
Accepted Dec 3, 2017 \\
\hline
\end{tabular}

\section{Keyword:}

Control

Prevention

Rabies

Vaccine

Zoonosis

\begin{abstract}
Rabies is an acute viral infection of the central nervous system, caused by a lyssavirus in the family Rhabdoviridae. It is zoonotic viral disease that can affect all mammals, including humans, cats, dogs, and wildlife and farm animals. The virus is present in the saliva of affected animals, and the most frequent method of transmission to humans is by bites, scratches or licks to broken skin or mucous membranes. The disease has a long incubation period (six months) and symptoms may take several weeks to appear after infection. The first clinical symptom is neuropathic pain at the site of infection or wound due to viral replication. Diagnosis can only be confirmed by laboratory tests preferably conducted post mortem on central nervous system tissue removed from cranium. This paper reviews the possible prevention and control of rabies. Essential components of rabies prevention and control include ongoing public education, responsible pet ownership, routine veterinary care and vaccination, and professional continuing education. Control strategies include quarantine, confirmation of diagnosis, determining the origin and spread of an outbreak. Since rabies is invariably fatal and deadly viral disease that can only be prevented the collaborative effort between Veterinarians and human health care professionals are needed in the prevention and control of rabies.
\end{abstract}

Copyright @ 2017 Institute of Advanced Engineering and Science. All rights reserved.

\section{Corresponding Author:}

Nejash Abdela,

Jimma University, College of Agriculture and Veterinary Medicine,

School of Veterinary Medicine,

Jimma, Ethiopia.

Email: nejash.abdela@gmail.com

\section{INTRODUCTION}

Rabies is a viral disease that affects the central nervous system of warm-blooded animals, including humans [1],[2] and it is a zoonotic viral disease that produces almost invariably fatal encephalitis in humans and most other mammals [3]. The disease is characterized by the development of severe nervous symptoms that lead to paralysis and death [4]. Once symptoms of the disease develop, it is invariably fatal and deadly viral disease that can only be prevented but not cured [5]. Dogs remain the primary reservoir in developing countries, whereas wildlife species serve as hosts in developed nations [6].

Rabies is widely distributed throughout the world with the exception of Australia, New Zealand, Japan, a number of European countries and some Caribbean Islands. Wild animals serve as a large and mainly uncontrollable reservoir of sylvatic rabies, which is an increasing threat to the human population and to domestic animals in many countries [1]. Rabies is an acute viral infection of the central nervous system, caused by a lyssavirus in the family Rhabdoviridae [4],[7].

The name Rhabdo comes from the Greek and identifies the characteristic bullet or rod-shape of the viruses [1]. It affects all mammals, including humans, cats, dogs, wild life and farm animals. In animals, three forms are classically described; prodromal, excitement (furious) and paralytic (dumb). The virus is present in the saliva of affected animals, and the most frequent method of transmission to humans is by bites, scratches or licks to broken skin or mucous membranes [8]. The virus is spread through infected saliva in 
bites, scratches and through licks from infected animals in open wounds or on mucosal membranes [7],[9],[10].

Rabies infection in humans is still a major public health Problem all over the world [11]. Rabies kills an estimated 35,000 per year, mostly in Africa, Asia and Latin America [6]. The World Health Organization (WHO) considers rabies to be a neglected disease and declare it to be primarily a problem in areas troubled with poverty and with a lack of economic resources [12]. With over 55000 human deaths a year [7] and signs of it re-emerging [9]. The domestic dog is the most important vector of human exposure [13]. It is possible to prevent a person exposed to the virus from getting ill to rabies by neutralizing virus with antibodies before the virus invades the nervous tissue. This is done through vaccination and/or use of immunoglobulins, so called post-exposure prophylaxis (PEP) [7],[14]. Public awareness and an increase of knowledge about rabies disease, first aid measures after dog bites, increased knowledge about dog behavior and how to avoid getting bitten by dogs are suggested methods to prevent rabies in humans [7],[9]. Therefore the main objective of this paper is to review the current information available for prevention and control of rabies.

\section{LITERATURE REVIEW}

\subsection{Etiology}

The causative agent of rabies is a member of the Lyssavirus genus of the Rhabdoviridae family of bullet-shaped viruses, which have a single-stranded RNA genome [1],[7]. The genus includes the classical rabies virus (genotype 1) and six so-called rabies-related viruses, Lagos bat virus (genotype 2), Mokola virus (genotype 3), Duvenhage virus (genotype 4), European bat lyssaviruses 1 and 2 (genotypes 5 and 6), and the recently discovered Australian bat genotype 7 [15]. The genus Lyssa virus comprises rabies virus and closely related viruses, including Mokola virus, Lagos bat virus and Duvenhage virus from Africa, European bat virus 1 and 2 and Australian bat Lyssavirus. Each of these viruses is considered capable of causing rabies like disease in animals and humans [1]. Rabies virus can be inactivated by sodium hypochlorite, $45-75 \%$ ethanol, iodine preparations, quaternary ammonium compounds, formaldehyde, phenol, ether, trypsin, $\beta-$ propiolactone, and some other detergents. It is also inactivated by a very low $\mathrm{pH}$ (below 3 ) or very high $\mathrm{pH}$ (greater than 11). This virus is susceptible to ultraviolet radiation. It is rapidly inactivated by sunlight and drying, and (in dried blood and secretions) it does not survive for long periods in the environment [16].

\subsection{Epidemiology}

\subsection{1. geographic distribution}

With some exceptions (particularly islands), rabies virus is found worldwide. Some countries such as the United Kingdom, Ireland, Sweden, Norway, Iceland, Japan, Australia, New Zealand, Singapore, most of Malaysia, Papua New Guinea, the Pacific Islands and some Indonesian islands have been free of this virus for many years [17]. Rabies is a serious disease threat to humans, domestic animals, and wildlife. Worldwide rabies kills about 50,000 - 100,000 people/year and countless domestic and wild animals [18]. In Europe the red fox is the most important reservoir host and vector of rabies. An increase in incidence of rabies in foxes result in an increase in incidence of rabies in domestic animals such as cattle, sheep, horse, cat, dog and others.

Sylvatic and urban rabies cycles occur concurrently in some regions, while the sylvatic cycle predominates in others. For example, wild animals accounted for more than $90 \%$ of the animal rabies cases reported in the U.S. and Canada in 2010. Rabies can be a serious concern in some rare or endangered species. In Africa, the Ethiopian wolf (Canis simensis) and African wild dogs (Lycaon pictus) are threatened by this virus. Although cases of rabies tend to be sporadic, epizootics are possible [16].

\subsubsection{Host range}

All mammals are susceptible to rabies, but only a limited number of species also act as reservoir hosts. They include members of the families Canidae (dogs, jackals, coyotes, wolves, foxes and raccoon dogs), Mustelidae (e.g., skunks), Viverridae (e.g., mongooses), and Procyonidae (raccoons), and the order Chiroptera (bats) [17]. Rabies reservoirs are generally grouped into terrestrial (i.e., land-dwelling) species and bat species. Rabies can occur sporadically in individuals or can exist in an enzootic or epizootic state in animal populations. In enzootic state rabies is indigenous to a reservoir species in a locality and occurs with a relatively stable incidence rate. An epizootic occurs when the incidence of disease increases markedly in the reservoir species. Rabies that is transmitted sporadically from reservoir to non-reservoir species is said to "spillover". These reservoir species are: raccoon (Procyon lotor), striped skunk (Mephitis mephitis), coyote (Canis latrans; infected with the dog variant), gray fox (Urocyon cinereoargenteus), and Arctic fox (Alopex lagopus) and red fox (Vulpes vulpes) [18]. 


\subsubsection{Transmission}

A rabies exposure is any bite, scratch, or other situation in which saliva, cerebral spinal fluid, tears, or nervous tissue from a suspect or known rabid animal or person enters an open wound, is transplanted into, or comes in contact with mucous membranes of another animal or person. On rare occasions human rabies has been acquired by inhalation of airborne virus in laboratories working with live rabies virus and in caves with millions of bats. The common mode of transmission of rabies in man is by bite of a rabid animal or the contamination of scratch wounds by virus infected saliva [19].

\subsection{Pathogenesis}

Rabies virus enters the body through wounds or by direct contact with mucosal surfaces. It cannot cross intact skin. Rabies virus replicates in the bitten muscle (local viral proliferation in non-neural tissue) and gains access (viral attachment) to motor endplates and motor axons to reach the central nervous system [20]. Virions are carried in transport vesicles [21] and travel to the central nervous system (CNS) exclusively by fast retrograde transport along motor axons, with no uptake by sensory or sympathetic endings [22].

Following centrifugal transport along efferent cranial nerves, the salivary glands become infected and virus particles are shed in the saliva. Infection of the brain commonly leads to behavioural changes that induce the host to bite other animals, thereby transmitting the virus. The widespread central nervous system infection almost inevitably leads to death, usually through respiratory paralysis, but also through secondary circulatory, metabolic or infectious processes [23],[24].

Viruses can also enter motor axons in peripheral nerves directly during a penetrating injury. The incubation period varies from 5 days to several years (usually 2-3 months; rarely more than 1 year), depending on the amount of virus in the inoculum, the density of motor endplates at the wound site and the proximity of virus entry to the central nervous system [25].

The incubation period is less than 50 days if the patient is bitten on the head or neck or if a heavy inoculum is transferred through multiple bites, deep wounds, or large wounds. A person with a scratch on the hand may take longer to develop symptoms of rabies than a person who receives a bite to the head. In dogs and cats, the incubation period is 10 days to 6 months; most cases become apparent between 2 weeks and 3 months. In cattle, an incubation period from 25 days to more than 5 months has been reported in vampire battransmitted rabies. In humans, the incubation period can be a few days to several years. Most cases become apparent after 1-3 months [17].

\subsection{Clinical signs}

The clinical picture can be highly variable between different species, individuals of the same species, and even within the course of the disease in a particular individual. As the disease progresses, animals with rabies may show strange behavior. Any clinical suspicion of rabies must be confirmed by laboratory examination [5].

The initial clinical signs are often nonspecific and may include fearfulness, restlessness, anorexia or an increased appetite, vomiting, diarrhea, a slight fever, dilation of the pupils, hyperreactivity to stimuli and excessive salivation. The first sign of post-vaccinal rabies is usually lameness in the vaccinated leg. Animals often have behavioral and temperament changes, and may become either unusually aggressive or uncharacteristically affectionate [17].

\subsubsection{Prodromal stage}

After a certain incubation period, the onset of clinical symptoms follows. During this first stage which usually lasts for about 1-3 days minor behavioral changes might occur, i.e. aggressiveness in tame animals, daytime activities in nocturnal animals, no fear of humans in wild animals or abnormalities in appetite [26].

\subsubsection{Excitement (furious) phase}

Eventually, the prodromal stage is followed by a period of severe agitation and aggressiveness. The animal often bites any material. Rabid dogs, for example, may develop a typical high barking sound during furious rabies. Death may follow convulsions even without the paralytic stage of the disease [17].

The furious form is characterized by restlessness, wandering, howling, polypnea, drooling and attacks on other animals, people or inanimate objects. Affected animals often swallow foreign objects such as sticks and stones. Wild animals frequently lose their fear of humans, and may attack humans or animal species they would normally avoid (e.g., porcupines). Nocturnal animals may be visible during the day. In cattle, unusual alertness can also be a sign of this form [17]. 


\subsubsection{Paralytic (dumb) phase}

The "dumb" form of rabies is characterized by progressive paralysis. In this form, the throat and masseter muscles become paralyzed; the animal may be unable to swallow, and it can salivate profusely. Laryngeal paralysis can cause a change in vocalization, including an abnormal bellow in cattle or a hoarse howling in dogs. There may also be facial paralysis or the lower jaw may drop. Ruminants may separate from the herd and can become somnolent or depressed. Rumination may stop. Ataxia, incoordination and ascending spinal paresis or paralysis are also seen [17]. This stage is characterized by the inability to swallow, leading to a typical sign of foaming saliva around the mouth. Some animals may develop paralysis beginning at the hind extremities. Eventually, complete paralysis is followed by death [26].

\subsection{Diagnosis}

Laboratory diagnosis of rabies in humans and animals is essential for timely post-exposure prophylaxis. Rabies diagnosis may be carried out either in vivo or postmortem [27]. Infection with rabies virus can be difficult to diagnose ante-mortem. Although hydrophobia is highly suggestive, no clinical signs of disease are pathognomonic for rabies. Historical reliance on the detection of accumulations of Negribodies is no longer regarded as suitable for diagnostic assessment because of low sensitivity and alternative laboratory-based tests based have been developed to conclusively confirm infection [4].

Most diagnostic tests for rabies virus in animals need brain material for diagnosis and as such are often only possible post mortem [28]. The diagnosis of rabies in animals can be made by taking any part from the affected brain. But in order to rule out rabies, the test must include tissues from at least two locations in brain, from the brain stem and cerebellum. There are many diagnosis methods for detection of rabies in animals like (Table 1); direct florescent antibody, mouse inoculation technique, tissue culture infection technique, and polymerase chain reaction [29]. Brain samples are most readily taken by breaching the skull and sampling directly. Brain smears or touch impressions are used for the meni detection of virus antigen with the fluorescent antibodytest (FAT) for both human and animal samples. In animals the direct fluorescent antibody test (dFAT) is the recommended diagnostic test. This test detects the presence of rabies antigens in brain tissue. Other diagnostic techniques include reverse transcription polymerase chain reaction (RT-PCR), direct rapid neur immunohistochemistry test (dRIT) and serological tests (Fluorescent antibody neutralization test, rapid pres fluorescent focus inhibition test). In humans, the rabies recommended test is dFAT on brain tissue. Other diagnostic tests that have been used are RT-PCR and dRIT [4].

Clinical diagnosis of rabies divided upon three stages in human; prodromal, excitement (furious) and paralytic (dumb). But all these stages cannot be observed in an individual. The very first clinical symptom is neuropathic pain at the site of infection or wound due to viral replication. Following by the prodromal phase either or both the excitement or paralytic forms of the disease may be observed in the particular species. It is also documented that cats are more likely to develop furious rabies than dogs [29]. In some cases, no signs are observed and rabies virus has been identified as the case of sudden death [30]. Diagnosis can only be confirmed by laboratory tests preferably conducted post mortem on central nervous system tissue removed from cranium [31].

Rabies must be considered in the differential diagnosis of any suspected mammalian meningitis/ encephalitis, distemper, infectious canine hepatitis and cerebral cysticercosis (Taenia solium) in dogs, sporadic bovine encephalomyelitis (Chlamydia psittaci), heartwater in cattle and sheep. Other conditions like mineral/ pesticide poisoning and Plant poisoning from Pennisetum clandestinum (kikuyu grass) in cattle, Cynanchum spp (monkey rope) in sheep should be considered [23].

Table 1. Diagnostic Techniques for Rabies

\begin{tabular}{lll}
\hline \multicolumn{1}{c}{ Technique } & \multicolumn{1}{c}{ Specimen } & \multicolumn{1}{c}{ Advantage/Disadvantage } \\
\hline $\begin{array}{l}\text { Direct Fluorescent Antibody } \\
\text { Technique (DFA) }\end{array}$ & $\begin{array}{l}\text { Target organs, such as brain, salivary glands, } \\
\text { liver, spleen, pancreas, nuchal skin, brain is the } \\
\text { most appropriate sample }\end{array}$ & $\begin{array}{l}\text { Applicable with most tissue sources. Not } \\
\text { applicable in decomposed tissue }\end{array}$ \\
$\begin{array}{l}\text { Mouse Inoculation } \\
\text { Technique (MIT) }\end{array}$ & $\begin{array}{l}\text { Similar to DFA } \\
\begin{array}{l}\text { Tissue Culture } \\
\text { technique (TCIT) }\end{array}\end{array}$ & Similar to DFA \\
$\begin{array}{l}\text { Polymerase Chain Reaction } \\
\text { (PCR) }\end{array}$ & $\begin{array}{l}\text { Similar to DFA including body fluids, saliva, } \\
\text { urine, CSF }\end{array}$ & $\begin{array}{l}\text { Only use fresh tissues } \\
\text { Axpensive in all tissue conditions }\end{array}$ \\
\hline
\end{tabular}

Source: (Yousaf et al., 2012) [29] 


\subsection{Prevention and control}

There is no certain cure for rabies except supportive care. Rabies can be prevented before the latent symptoms can develop, consists of giving a person an injection of rabies immune globulin and another injection of rabies vaccine as soon as possible after the bite or exposure to saliva from an infected animal [29].

\subsubsection{Principles of rabies prevention and control}

\subsubsection{Case definition}

An animal is determined to be rabid after diagnosis by a qualified laboratory and confirmation either by a positive direct fluorescent antibody test (preferably performed on central nervous system tissue) or Isolation of rabies virus in cell culture or in a laboratory animal.

\subsubsection{Rabies exposure}

Rabies is transmitted when the virus is introduced into bite wounds, open cuts in skin, or onto mucous membranes from saliva or other potentially infectious material such as neural tissue [32].

\subsubsection{Public health education}

Essential components of rabies prevention and control include ongoing public education, responsible pet ownership, routine veterinary care and vaccination, and professional continuing education. The majority of animal and human exposures to rabies can be prevented by raising awareness concerning: rabies transmission routes, and avoiding contact with wildlife. Prompt recognition and reporting of possible exposures to medical professionals and local public health authorities is critical [32].

\subsubsection{Human rabies prevention}

Human rabies can be prevented by a) eliminating exposure to rabies virus, b) providing appropriate rabies pre-exposure prophylaxis, and c) prompt local treatment of bite wounds combined with appropriate rabies post-exposure prophylaxis [32]. Inactivated human vaccines are available for at risk veterinary staff, other animal handlers, wildlife officers, laboratory workers and others at high risk of exposure.

\subsubsection{Mestic animal rabies control}

The primary components of a rabies control program for companion animals are: immunization and licensing; stray animal control; reporting, investigation, and isolation of animals involved in bite incidents; and public education. Multiple vaccines are licensed for use in domestic animal species. Vaccines available include: inactivated or modified live virus vectored products; products for intramuscular and subcutaneous administration; products with durations of immunity from one to 4 years; and products with varying minimum age of vaccination [32].

\subsubsection{Animal bites reporting}

The local health officer or designee shall be immediately notified of any person or animal bitten by or potentially exposed to a rabid or suspected rabid animal. In addition, the local health officer or designee shall be notified when any person is bitten by a mammal. Potential human rabies exposures are then evaluated and rabies post-exposure prophylaxis (PEP) recommendations made [32].

\subsubsection{Stray animals}

Stray dogs, cats, and ferrets should be removed from the community. Local health departments and animal control officials can enforce the removal of strays more effectively if owned animals are required to have identification and are confined or kept on leash. Strays should be impounded for at least 3 business days to determine if human exposure has occurred and to give owners sufficient time to reclaim animals [33].

\subsubsection{Isolation of animals exposed to rabies}

Any animal bitten by, scratched by, or having direct contact with a wild mammal that is not available for rabies testing should be regarded as having been exposed to rabies. All livestock species-horses, cattle, sheep, goats, llamas/alpacas, and swine are susceptible to rabies infection. Cattle and horses are the livestock species most frequently diagnosed with rabies. Unvaccinated livestock bitten by or exposed to a rabid or suspect rabid animal should be euthanized [32].

\subsubsection{Wild animal rabies control}

Principles of rabies prevention should focus on excluding wild animals from areas of human and domestic animal habitation and activity, and avoidance of contact with possibly rabid wild animals. Public 
education on the risks of rabies transmission from wild animals is paramount to effective disease prevention. Immunization of wildlife by widespread distribution of vaccine-impregnated oral baits has shown variable success toward arresting the propagation of rabies in raccoons and coyotes in other states. The use of oral rabies vaccines (ORV) for the mass vaccination of free-ranging wildlife should be considered in selected situations [32].

\subsubsection{Animal pre-exposure vaccination}

A number of recently developed, highly-effective, thermo stable, inactivated vaccines are available for veterinary use. The duration of immunity conferred varies from one to three years. Most veterinary vaccines are only registered for use in specific species, for example dogs. All rabies vaccines registered for human and animal use must conform to established potency standards. A minimum antigenic potency of 2.5 IU per dose is mandatory [34].

The vaccines may be used in young pups, but they must be boosted at three months of age and again within the following year. Revaccination must be carried out every three years thereafter. Cattle and sheep may be vaccinated annually or every two to three years, depending on the vaccine manufacturer's instructions. Following an outbreak in domestic livestock, vaccination of animals without visible bite wounds is strongly recommended [35].

There are currently 17 parenteral animal rabies vaccines licensed by the U.S. Department of Agriculture (USDA) for use in dogs, cats, sheep, cattle, horses, and/or ferrets. Some are approved for dogs or cats for a multi-year immunity period and others for only a 1-year period. All dogs and cats should be revaccinated 12 months after initial vaccination regardless of the length of immunity period of the initial vaccine. Obtaining a booster vaccination immediately following an exposure to a rabid animal is important to ensure adequate protection against the virus [35].

\subsubsection{Animal post-exposure treatment}

Findings from a study conducted by Hanlon .et al., 2002 suggested that 5 doses of canine rabies vaccine administered on days $0,3,14,21$ and 35 along with murine anti-rabies antibody on day 0 may be effective in protecting a previously unvaccinated animal exposed to rabies. Regardless of the age of the animal at initial vaccination, a booster vaccination should be administered 1 year later [5]. If signs suggestive of rabies develop (e.g., paralysis, seizures, etc.), the animal should be euthanized and the head shipped for testing [36].

\subsubsection{Outbreak prevention and control}

The emergence of new rabies virus variants or the introduction of non-indigenous viruses poses a significant risk to humans, domestic animals, and wildlife [37]. A rapid and comprehensive response includes the following measures [38]:

$\checkmark$ Characterize the virus at the national reference laboratory.

$\checkmark$ Identify and control the source of the introduction.

$\checkmark$ Enhance laboratory-based surveillance in wild and domestic animals.

$\checkmark$ Increase animal rabies vaccination rates.

$\checkmark$ Restrict the movement of animals.

$\checkmark$ Evaluate the need for vector population reduction.

$\checkmark$ Coordinate a multiagency response.

$\checkmark$ Provide public and professional outreach and education.

\subsubsection{Rabies control strategies}

According to Bishop et al., (2003) Rabies Control Strategies includes quarantine, confirmation of diagnosis, determining the origin and spread of an outbreak, and specific measures to terminate transmission [23]. It is absolutely essential to confirm the diagnosis of rabies. When an outbreak has been confirmed, the responsible state veterinarian decides on the area requiring vaccination.

\subsubsection{Large-scale vaccination}

Large-Scale Vaccination is the initial response to rabies outbreaks in endemic high-risk areas. The aim should be to vaccinate at least $70 \%$ of the animal population at risk in a single campaign within as short a period as possible. Where this cannot be achieved, two or more campaigns should be conducted within a year. 


\subsubsection{Cordon vaccination}

The aim of cordon vaccination is to create a barrier of animal immunity. This approach has become increasingly important during the second phase of canine rabies elimination, particularly in the vicinity of international borders and national as well as provincial game reserves. This is necessary to prevent reinfection of areas currently free from rabies and to protect rabies-free wildlife areas. A cordon of 20 to $30 \mathrm{~km}$ is usually sufficient for this purpose

\subsubsection{Ring vaccination}

In areas where an isolated case has been diagnosed, ring vaccination with a radius of 20 to 30 $\mathrm{km}$, where at least $70 \%$ of dogs are vaccinated, has proven successful in controlling or eliminating rabies

\subsubsection{Door-to-door vaccination}

Vaccination on a home-to-home basis is generally more expensive, resource intensive and time consuming, but coverage is invariably better. An example of the success of this approach is illustrated by a campaign in Eerstehoek, a rural district in Mpumalanga. In 1998, 20 officials vaccinated 6498 dogs in 30 villages extending over $594 \mathrm{~km}^{2}$ on a home-to-home basis. All vaccinated dogs were identified with neckbands.

\subsubsection{Central-point vaccination}

These campaigns are usually conducted at least annually in areas where rabies is endemic and the prevalence of outbreaks is high. The public are requested to bring their pets to identified venues. Accurate epidemiological information, good advertising and adequate planning are of paramount importance to ensure high coverage. Results vary from 20 to $80 \%$ and depend on the degree of attention to detail. Coverage is generally better if the campaign follows shortly after a well-publicised rabies outbreak.

\subsubsection{Oral vaccination}

Sylvatic rabies has been successfully eradicated in Switzerland and other European countries using bait vaccine. A bait containing a live, avirulent rabies virus mutant, SAG2, has been formulated specifically for dogs and the first field trials were conducted in South Africa.

\section{CONCLUSIONS AND RECOMMENDATIONS}

Rabies is a fatal viral zoonotic disease and a serious public health problem. Though its occurrence is worldwide, it is important in developing countries like Ethiopia. This is because of widespread occurrence of large number of stray dogs. In Africa, the Ethiopian wolf (Canis simensis) and African wild dogs (Lycaon pictus) are threatened by this virus. It is transmitted only when the virus is introduced in to bite wound or mucus membrane. The disease is incurable but preventable. In animals, rabies prevention is based on vaccination and the avoidance of contact with infected animals. The majority of animal and human exposures to rabies can be prevented by raising public awareness on rabies transmission routes, and avoiding contact with wildlife. Based on the above conclusions the following recommendations could be forwarded.

Raising awareness of the community on the mode of transmission, prevention and control of rabies is of paramount importance. Use of international law to support the control of communicable diseases. Regulations for stray dog management should be adopted and Strict control of free-ranging dogs and mandatory rabies vaccination should be enforced.

\section{ACKNOWLEDGEMENTS}

Above of all we would like to thanks almighty God for guiding us in every day of our life in every direction. Next we would like to acknowledge Jimma University College of Agriculture and Veterinary Medicine, and school of veterinary medicine for their facility support and internet access.

\section{REFERENCES}

[1] N. Moges, "Epidemiology, Prevention and Control Methods of Rabies in Domestic Animals: Review Article," European Journal of Biological Sciences, vol/issue: 7(2), pp. 85-90, 2015.

[2] O. G. Richard, et al., "A Review on Human Deaths Associated with Rabies in Nigeria," Journal of Vaccines \& Vaccination, 2015. 
[3] Black T. P. and Rupprecht C. E., "Rhabdoviruses," in Mandell G. L., et al., "Principles and Practice of Infectious Diseases," Philadelphia, ELSEVIER Churchill Livingstone, pp. 2047-56, 2004.

[4] E. Abera, et al., "Review on Rabies, with Emphasis on Disease Control and Eradication Measures," International Journal of Basic and Applied Virology, vol/issue: 4(2), pp. 60-70, 2015.

[5] Blackmore C., "Rabies Prevention and Control in Florida, Division of Disease Control and Health Protection, pp. $122,2014$.

[6] C. E. Rupprecht, et al., "Can rabies be eradicated?” Developments in biologicals, vol. 131, pp. 95-121, 2007.

[7] M. Nilsson, "Effect on rabies education program on rabies awareness, attitudes towards dogs and animal welfare among children in Lilongwe, Malawi," Epsilon, Examensarbete, pp. 26, 2014.

[8] Jackson A. C., "Why does the prognosis remain so poor in human rabies?" Expert. Rev. Anti Infect. Ther., vol. 8, pp. 623-625, 2010.

[9] S. J. Depani, et al., "Case report: Evidence of Rise in Rabies Cases in Southern Malawi - Better Preventative Measures Are Urgently Required,” Malawi medical Journal, vol/issue: 24(3), pp. 61-64, 2012.

[10] World Health Organization, "FAQs Frequently asked questions on rabies," New Delhi, India, 2013.

[11] American Public Health Association, APHA, "Rabies," in Heymann D., "Control of communicable diseases manual," Washington, pp. 498-508, 2008.

[12] World Health Organization, "Rabies," 2013. http://www.who.int/mediacentre/factsheets/fs099/en/index.html.

[13] Cleaveland S., et al., "Estimating human rabies mortality in the United Republic of Tanzania from dog bite injuries," Bulletin of the WHO, vol. 80, pp. 304-10, 2002.

[14] N. Permpalung, et al., "Trend of human rabies prophylaxis in developing countries: Toward optimal rabies immunization," Vaccine, vol. 31, pp. 4079-4083, 2013.

[15] M. Warrell, "Rabies encephalitis and its prophylaxis," Practical Neurology, vol/issue: 1(1), pp. 14-29, 2001.

[16] Center for Food Security and Public Health, CFSPH, "Iowa State University, Inistitute for International Cooperation in Animal Biologics," Rabies and Rabies-Related Lyssaviruses, 2012.

[17] World Organization for Animal Health [OIE], "Manual of diagnostic tests and vaccines for terrestrial animals," OIE, Rabies, 2008.

[18] Bruce C. and Margaret A. W., “Biological Resource Management Division,” Fort Collins, pp. 15-27, 2001.

[19] Morbidity and Mortality Weekly Report, MMWR, "Compendium of Animal Rabies Prevention and Control: Recommendations and Reports, vol/issue: 60(6), pp. 1-17, 2011.

[20] Ugolini G., "Use of rabies virus as a transneuronal tracer of neuronal connections: Implications for the understanding of rabies pathogenesis," Dev. Biols.(Basel), vol. 131, pp. 493-506, 2008.

[21] Klingen Y., et al., "Double-labeled rabies virus: live tracking of enveloped virus transport," J.Vir., vol. 82, pp. 237-245, 2008.

[22] Hemachudha T., "Human rabies: neuropathogenesis, diagnosis and management," Lancet Neur., vol. 12, pp. 498-513, 2013.

[23] G. C. Bishop, et al., "Speare and the Rabies Advisory Group," Rabies, Guide For The Medical, Veterinary and Allied Professions $2^{\text {nd }}$ ed, Government Printer, Pretoria, pp. 26, 2013.

[24] A. Shite, et al., "Challenges of Rabies," International Journal of Basic and Applied Virology, vol/issue: 4(2), pp. 41-52, 2015.

[25] Ugolini G., "Rabies virus as a transneuronal tracer of neuronal connections," Adv.Virs. Res., vol. 79, pp. 165-202, 2011.

[26] WHO, "Information Surveillance Report," Collaborating Centre for Rabies Surveillance and Research, Greifswald - Insel Riems, Germany, J.Rab.Bull.Eur.,vol. 37, pp. 2, 2013.

[27] C. A. Consales and V. L. Bolzan, "Rabies review: immunopathology, clinical aspects and treatment," Journal of Venomous Animals and Toxins including Tropical Diseases, vol/issue: 13(1), pp. 5-38, 2007.

[28] A. R. Fooks, et al., "Molecular tools for rabies diagnosis in animals," in Fooks A. R. and Müller T., Compendium of the OIE Global Conference on Rabies Control, pp. 75-87, 2012.

[29] M. Yousaf, et al., "Rabies molecular virology, diagnosis, prevention and treatment," Virology journal, vol/issue: 9(1), pp. 1, 2012.

[30] Boonlert L., "Laboratory Techniques for Rabies Diagnosis in Animals at QSMI," Thai. Red Cross Society J. Med. Assoc. Thai., vol. 88, pp. 550-553, 2005.

[31] Elhinney M., et al., "Diagnostic tools for the detection of rabies virus," EJCAP, vol. 18, pp. 03, 2008.

[32] California Department of Public Health, CDPH, Veterinary Public Health Section, "Compendium of Rabies Control and Prevention," pp. 1-17, 2012.

[33] Catherine M. and Brown, "Compendium of Animal Rabies Prevention and Control Committee Compendium of Animal Rabies Prevention and Control," 2011.

[34] World Health Organization, "Expert Committee on Rabies,” Technical Report; series 824, Geneva, 1992.

[35] Murray K. O., et al., "Rabies in vaccinated dogs and cats in the United States, 1997-2001," JAVMA, vol. 235, pp. 691-95, 2009.

[36] Hanlon C. A., et al., "Post-exposure prophylaxis for prevention of rabies in dogs," Am. J. Vet. Res., vol. 63, pp. 1096-110, 2002.

[37] Leslie M. J., et al., "Bat-associated rabies virus in skunks,” Emerg Infect Dis., vol. 12, pp. 1274-7, 2006.

[38] Rupprecht C. E., et al., "Control and prevention of rabies in animals: paradigm shifts," Dev Biol (Basel), vol. 125, pp. 103-11, 2006. 